\title{
Lower Bound for the Size of Maximal Nontraceable Graphs*
}

\author{
Marietjie Frick, Joy Singleton \\ University of South Africa, \\ P.O. Box 392, Unisa, 0003, \\ South Africa. \\ e-mail: frickm@unisa.ac.za singlje@unisa.ac.za
}

Submitted: Jun 25, 2004; Accepted: Jul 4, 2005; Published Jul 19, 2005

2000 Mathematics Subject Classification: 05C38

\begin{abstract}
Let $g(n)$ denote the minimum number of edges of a maximal nontraceable graph of order $n$. Dudek, Katona and Wojda (2003) showed that $g(n) \geq\left\lceil\frac{3 n-2}{2}\right\rceil-2$ for $n \geq 20$ and $g(n) \leq\left\lceil\frac{3 n-2}{2}\right\rceil$ for $n \geq 54$ as well as for $n \in I=\{22,23,30,31,38,39,40,41,42$, $43,46,47,48,49,50,51\}$. We show that $g(n)=\left\lceil\frac{3 n-2}{2}\right\rceil$ for $n \geq 54$ as well as for $n \in I \cup\{12,13\}$ and we determine $g(n)$ for $n \leq 9$.
\end{abstract}

Keywords: maximal nontraceable, hamiltonian path, traceable, nontraceable, nonhamiltonian

\section{Introduction}

We consider only simple, finite graphs $G$ and denote the vertex set, the edge set, the order and the size of $G$ by $V(G), E(G), v(G)$ and $e(G)$, respectively. The open neighbourhood of a vertex $v$ in $G$ is the set $N_{G}(v)=\{x \in V(G): v x \in E(G)\}$. If $U$ is a nonempty subset of $V(G)$ then $\langle U\rangle$ denotes the subgraph of $G$ induced by $U$.

A graph $G$ is hamiltonian if it has a hamiltonian cycle (a cycle containing all the vertices of $G$ ), and traceable if it has a hamiltonian path (a path containing all the vertices of $G$ ). A graph $G$ is maximal nonhamiltonian (MNH) if $G$ is not hamiltonian, but $G+e$ is hamiltonian for each $e \in E(\bar{G})$, where $\bar{G}$ denotes the complement of $G$. A graph $G$ is maximal nontraceable (MNT) if $G$ is not traceable, but $G+e$ is traceable for each $e \in E(\bar{G})$.

${ }^{*}$ This material is based upon research for a thesis at the University of South Africa and is supported by the National Research Foundation under Grant number 2053752. 
In 1978 Bollobás [1] posed the problem of finding the least number of edges, $f(n)$, in a MNH graph of order $n$. Bondy [2] had already shown that a MNH graph with order $n \geq 7$ that contained $m$ vertices of degree 2 had at least $(3 n+m) / 2$ edges, and hence $f(n) \geq\lceil 3 n / 2\rceil$ for $n \geq 7$. Combined results of Clark, Entringer and Shapiro [3], [4] and Lin, Jiang, Zhang and Yang [7] show that $f(n)=\lceil 3 n / 2\rceil$ for $n \geq 19$ and for $n=6,10,11,12,13,17$. The values of $f(n)$ for the remaining values of $n$ are also given in $[7]$.

Let $g(n)$ denote the minimum number of edges in a MNT graph of order $n$. Dudek, Katona and Wojda [5] proved that

$$
g(n) \geq\left\lceil\frac{3 n-2}{2}\right\rceil-2 \text { for } n \geq 20
$$

and showed, by construction, that

$$
g(n) \leq\left\lceil\frac{3 n-2}{2}\right\rceil \text { for } n \geq 54
$$

as well as for $n \in I=\{22,23,30,31,38,39,40,41,42,43,46,47,48,49,50,51\}$.

We prove, using a method different from that in [5], that

$$
g(n) \geq\left\lceil\frac{3 n-2}{2}\right\rceil \text { for } n \geq 10 .
$$

We also construct graphs of order $n=12,13$ with $\left\lceil\frac{3 n-2}{2}\right\rceil$ edges and thus show that

$$
g(n)=\left\lceil\frac{3 n-2}{2}\right\rceil \text { for } n \geq 54 \text { as well as for } n \in I \cup\{12,13\} .
$$

We also determine $g(n)$ for $n \leq 9$.

\section{Auxiliary Results}

In this section we present some results concerning MNT graphs, which we shall use, in the next section, to prove that a MNT graph of order $n \geq 10$ has at least $\frac{3 n-2}{2}$ edges. The first one concerns the lower bound for the number of edges of MNH graphs. It is the combination of results proved in [2] and [7].

Theorem 1 (Bondy and Lin, Jiang, Zhang and Yang) If G is a MNH graph of order n, then $e(G) \geq \frac{3 n}{2}$ for $n \geq 6$.

The following lemma, which we proved in [6], will be used frequently.

Lemma 2 Let $Q$ be a path in a MNT graph $G$. If $\langle V(Q)\rangle$ is not complete, then some internal vertex of $Q$ has a neighbour in $G-V(Q)$. 
Proof. Let $u$ and $v$ be two nonadjacent vertices of $Q$. Then $G+u v$ has a hamiltonian path $P$. Let $x$ and $y$ be the two endvertices of $Q$ and suppose no internal vertex of $Q$ has a neighbour in $G-V(Q)$. Then $P$ has a subpath $R$ in $\langle V(Q)\rangle+u v$ and $R$ has either one or both endvertices in $\{x, y\}$. If $R$ has only one endvertex in $\{x, y\}$, then $P$ has an endvertex in $Q$. In either case the path obtained from $P$ by replacing $R$ with $Q$ is a hamiltonian path of $G$.

The following lemma is easy to prove.

Lemma 3 Suppose $T$ is a cutset of a connected graph $G$ and $A_{1}, \ldots, A_{k}$ are components of $G-T$.

(a) If $k \geq|T|+2$, then $G$ is nontraceable.

(b) If $G$ is $M N T$ then $k \leq|T|+2$.

(c) If $G$ is $M N T$ and $k=|T|+2$, then $\left\langle T \cup A_{i}\right\rangle$ is complete for $i=1,2, \ldots, k$.

Proof. (a) and (b) are obvious. If (c) is not true, then there is an $i$ such that $\left\langle T \cup A_{i}\right\rangle$ has two nonadjacent vertices $x$ and $y$. But then $T$ is a cutset of the graph $G+x y$ and $(G+x y)-T$ has $|T|+2$ components and hence $G+x y$ is nontraceable, by (a).

The proof of the following lemma is similar to the previous one.

Lemma 4 Suppose $B$ is a block of a connected graph $G$.

(a) If $B$ has more than two cut-vertices, then $G$ is nontraceable.

(b) If $G$ is $M N T$, then $B$ has at most three cut-vertices.

(c) If $G$ is $M N T$ and $B$ has exactly three cut-vertices, then $G$ consists of exactly four blocks, each of which is complete.

In [6] we proved some results concerning the degrees of the neighbours of the vertices of degree 2 in a 2-connected MNT graph, which enabled us to show that the average degree of the vertices in a 2-connected MNT graph is at least 3. We now restate those results in a form that is applicable also to MNT graphs which are not 2-connected. (Note that in a 2-connected graph no two vertices of degree 2 are adjacent to one another.)

Lemma 5 If $G$ is a connected MNT graph and $v \in V(G)$ with $d(v)=2$, then the neighbours of $v$ are adjacent. Also, one of the neighbours has degree at least 4 and the other neighbour has degree 2 or at least 4.

Proof. Let $N_{G}(v)=\left\{x_{1}, x_{2}\right\}$ and let $Q$ be the path $x_{1} v x_{2}$. Since $N_{G}(v) \subseteq Q$, it follows from Lemma 2 that $\langle V(Q)\rangle$ is a complete graph; hence $x_{1}$ and $x_{2}$ are adjacent.

Since $G$ is connected and nontraceable, at least one of $x_{1}$ and $x_{2}$ has degree bigger that 2. Suppose $d\left(x_{1}\right)>2$ and let $z \in N\left(x_{1}\right)-\left\{v, x_{2}\right\}$. If $Q$ is the path $z x_{1} v x_{2}$ then, since $d(v)=2$, the graph $\langle V(Q)\rangle$ is not complete and hence it follows from Lemma 2 that $d\left(x_{1}\right) \geq 4$. Similarily if $d\left(x_{2}\right)>2$, then $d\left(x_{2}\right) \geq 4$. 
Lemma 6 Suppose $G$ is a connected MNT graph with distinct nonadjacent vertices $v_{1}$ and $v_{2}$ such that $d\left(v_{1}\right)=d\left(v_{2}\right)=2$.

(a) If $v_{1}$ and $v_{2}$ have exactly one common neighbour $x$, then $d(x) \geq 5$.

(b) If $v_{1}$ and $v_{2}$ have the same two neighbours $x_{1}$ and $x_{2}$, then $N_{G}\left(x_{1}\right)-\left\{x_{2}\right\}=$ $N_{G}\left(x_{2}\right)-\left\{x_{1}\right\}$ and $d\left(x_{1}\right)=d\left(x_{2}\right) \geq 5$.

Proof. (a) Let $N\left(v_{i}\right)=\left\{x, y_{i}\right\} ; i=1$, 2. It follows from Lemma 5 that $x$ is adjacent to $y_{i} ; i=1,2$. Let $Q$ be the path $y_{1} v_{1} x v_{2} y_{2}$. Since $\langle V(Q)\rangle$ is not complete, it follows from Lemma 2 that $x$ has a neighbour in $G-V(Q)$. Hence $d(x) \geq 5$.

(b) From Lemma 5 it follows that $x_{1}$ and $x_{2}$ are adjacent. Let $Q$ be the path $x_{2} v_{1} x_{1} v_{2}$. $\langle V(Q)\rangle$ is not complete since $v_{1}$ and $v_{2}$ are nonadjacent. Thus it follows from Lemma 2 that $x_{1}$ has a neighbour in $G-V(Q)$. Now suppose $p \in N_{G-V(Q)}\left(x_{1}\right)$ and $p \notin N_{G}\left(x_{2}\right)$. Then a hamiltonian path $P$ in $G+p x_{2}$ contains a subpath of either of the forms given in the first column of Table 1 . Note that $i, j \in\{1,2\} ; i \neq j$ and that $L$ represents a subpath of $P$ in $G-\left\{x_{1}, x_{2}, v_{1}, v_{2}, p\right\}$. If each of the subpaths is replaced by the corresponding subpath in the second column of the table we obtain a hamiltonian path $P^{\prime}$ in $G$, which leads to a contradiction.

\begin{tabular}{|l|l|}
\hline Subpath of $P$ & Replace with \\
\hline$v_{i} x_{1} v_{j} x_{2} p$ & $v_{i} x_{2} v_{j} x_{1} p$ \\
\hline$v_{i} x_{1} L p x_{2} v_{j}$ & $v_{i} x_{2} v_{j} x_{1} L p$ \\
\hline
\end{tabular}

Table 1

Hence $p \in N_{G}\left(x_{2}\right)$. Thus $N_{G}\left(x_{1}\right)-\left\{x_{2}\right\} \subseteq N_{G}\left(x_{2}\right)-\left\{x_{1}\right\}$. Similarly $N_{G}\left(x_{2}\right)-\left\{x_{1}\right\} \subseteq$ $N_{G}\left(x_{1}\right)-\left\{x_{2}\right\}$. Thus $N_{G}\left(x_{1}\right)-\left\{x_{2}\right\}=N_{G}\left(x_{2}\right)-\left\{x_{1}\right\}$ and hence $d\left(x_{1}\right)=d\left(x_{2}\right)$. Now let $Q$ be the path $p x_{1} v_{1} x_{2} v_{2}$. Since $\langle V(Q)\rangle$ is not complete, it follows from Lemma 2 that $x_{1}$ or $x_{2}$ has a neighbour in $G-V(Q)$. Hence $d\left(x_{1}\right)=d\left(x_{2}\right) \geq 5$.

Lemma 7 Suppose $G$ is a connected $M N T$ graph of order $n \geq 6$ and that $v_{1}, v_{2}$ and $v_{3}$ are vertices of degree 2 in $G$ having the same neighbours, $x_{1}$ and $x_{2}$. Then $G-\left\{v_{1}, v_{2}, v_{3}\right\}$ is complete and hence $e(G)=\frac{1}{2}\left(n^{2}-7 n+24\right)$.

Proof. The set $\left\{x_{1}, x_{2}\right\}$ is a cutset of $G$. Thus according to Lemma $3 G-\left\{v_{1}, v_{2}, v_{3}\right\}=$ $K_{n-3}$. Hence $e(G)=\frac{1}{2}(n-3)(n-4)+6$.

By combining the previous three results we obtain

Theorem 8 Suppose $G$ is a connected MNT graph without vertices of degree 1 or adjacent vertices of degree 2 . If $G$ has order $n \geq 7$ and $m$ vertices of degree 2 , then $e(G) \geq$ $\frac{1}{2}(3 n+m)$.

Proof. If $G$ has three vertices of degree 2 having the same two neighbours then, by Lemma $7, m=3$ and

$$
e(G)=\frac{1}{2}\left(n^{2}-7 n+24\right) \geq \frac{1}{2}(3 n+m) \text { when } n \geq 7 .
$$


We now assume that $G$ does not have three vertices of degree 2 that have the same two neighbours. Let $v_{1}, \ldots, v_{m}$ be the vertices of degree 2 in $G$ and let $H=G-\left\{v_{1}, \ldots, v_{m}\right\}$. Then by Lemmas 5 and 6 the minimum degree, $\delta(H)$ of $H$ is at least 3. Hence

$$
e(G)=e(H)+2 m \geq \frac{3}{2}(n-m)+2 m=\frac{1}{2}(3 n+m) .
$$

\section{The minimum size of a MNT graph}

Our aim is to determine the exact value of $g(n)$. By consulting the Atlas of Graphs [8], one can see, by inspection, that $g(2)=0, g(3)=1, g(4)=2, g(5)=4, g(6)=6$ and $g(7)=8$ (see Fig. 3).

We now give a lower bound for $g(n)$ for $n \geq 8$.

Theorem 9 If $G$ is a MNT graph of order $n$, then

$$
e(G) \geq \begin{cases}10 & \text { if } n=8 \\ 12 & \text { if } n=9 \\ \frac{3 n-2}{2} & \text { if } n \geq 10 .\end{cases}
$$

Proof. If $G$ is not connected, then $G=K_{k} \cup K_{n-k}$, for some positive integer $k<n$ and then, clearly, $e(G)>\frac{3 n-2}{2}$ for $n \geq 8$. Thus we assume that $G$ is connected.

We need to prove that the sum of the degrees of the vertices of $G$ is at least $3 n-2$. In view of Theorem 8 , we let

$$
M=\{v \in V(G) \mid d(v)=2 \text { and no neighbour of } v \text { has degree } 2\} .
$$

The remaining vertices of degree 2 can be dealt with simultaneously with the vertices of degree 1 . We let

$$
S=\{v \in V(G)-M \mid d(v)=2 \text { or } d(v)=1\} .
$$

If $S=\emptyset$, then it follows from Theorem 8 that $e(G) \geq \frac{1}{2}(3 n+m)$. Thus we assume that $S \neq \emptyset$.

We observe that, if $H$ is a component of the graph of $\langle S\rangle$, then either $H \cong K_{1}$ or $H \cong K_{2}$ and $N_{G}(H)-V(H)$ consists of a single vertex, which is a cut-vertex of $G$.

An example of such a graph $G$ is depicted in the figure below.

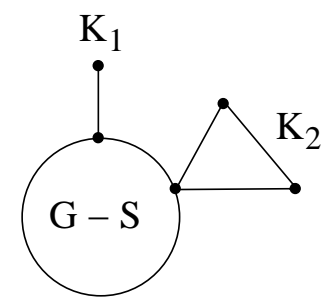

Fig. 1 
Let $s=|S|$. By Lemma 4 the graph $\langle S\rangle$ has at most three components. We thus have three cases:

CASE 1. $\langle S\rangle$ has exactly three components, say $H_{1}, H_{2}, H_{3}$ :

In this case the neighbourhoods of $H_{1}, H_{2}, H_{3}$ are pairwise disjoint; hence $G$ has three cut-vertices. Hence it follows from Lemma 4 that $G-S$ is a complete graph of order at least 3. Futhermore, for every possible value of $s$, the number of edges in $G$ incident with the vertices in $S$ is $2 s-3$. Thus

$$
e(G)=\left(\begin{array}{c}
n-s \\
2
\end{array}\right)+2 s-3 \text { for } s=3,4,5 \text { or } 6 ; s \leq n-3 .
$$

An easy calculation shows that, for each possible value of $s$,

$$
e(G) \geq \begin{cases}10 & \text { if } n=8 \\ 12 & \text { if } n=9 \\ \frac{3 n-2}{2} & \text { if } n \geq 10\end{cases}
$$

This case is a Zelinka Type II construction, cf. [9]. The graphs of smallest size of order 8 and 9 given by this construction are depicted in Fig. 3.

CASE 2. $\langle S\rangle$ has exactly two components, say $H_{1}, H_{2}$ :

In this case the number of edges in $G$ incident with the vertices in $S$ is $2 s-2$.

Subcase 2.1. $\quad N_{G}\left(H_{1}\right)=N_{G}\left(H_{2}\right)$ :

Then it follows from Lemma 3 that $G-S$ is a complete graph. Hence

$$
e(G)=\left(\begin{array}{c}
n-s \\
2
\end{array}\right)+2 s-2 \text { for } s=2,3 \text { or } 4 .
$$

Thus

$$
e(G) \geq \begin{cases}12 & \text { if } n=8 \\ 16 & \text { if } n=9 \\ \frac{3 n-2}{2} & \text { if } n \geq 10\end{cases}
$$

This case is a Zelinka Type I construction, cf. [9].

Subcase 2.2. $\quad N_{G}\left(H_{1}\right) \neq N_{G}\left(H_{2}\right)$ :

Let $N_{G}\left(H_{i}\right)=y_{i}, i=1,2$ and $y_{1} \neq y_{2}$.

If $y_{1} y_{2} \notin E(G)$ then $G+y_{1} y_{2}$ has a hamiltonian path $P$. But then $P$ has one endvertex in $H_{1}$ and the other in $H_{2}$ and contains the edge $y_{1} y_{2}$; hence $V(G-S)=\left\{y_{1}, y_{2}\right\}$. But then $G$ is disconnected. This contradiction shows that $y_{1} y_{2} \in E(G)$.

Now $G-S$ is not complete, otherwise $G$ would be traceable. Since $G+v w$, where $v$ and $w$ are nonadjacent vertices in $V(G-S)$, contains a hamiltonian path with one endvertex in $H_{1}$ and the other in $H_{2}$ and $y_{1} y_{2} \in E(G)$, it follows that $(G-S)+v w$ has 
a hamiltonian cycle. Hence $G-S$ is either hamiltonian or MNH. We consider these two cases separately:

Subcase 2.2.1. $\quad G-S$ is hamiltonian:

Then no hamiltonian cycle in $G-S$ contains $y_{1} y_{2}$, otherwise $G$ would be traceable. Thus $d_{G-S}\left(y_{i}\right) \geq 3$ for $i=1,2$.

It also follows from Lemma 3 that no vertex $v \in M$ can be adjacent to both $y_{1}$ and $y_{2}$ since the graph $\left\langle V\left(H_{i}\right) \cup T\right\rangle$, where $T=\left\{y_{1}, y_{2}\right\}$ is not complete, for $i=1,2$. If $v \in M$ is adjacent to to one of the $y_{i}$ 's for $i=1,2$, say $y_{1}$, then, since the neighbours of $v$ are adjacent, it follows that $d_{G-M-S}\left(y_{1}\right) \geq 3$.

It follows from our definition of $M$ and $S$ that $N_{G}(M) \cap S=\emptyset$. Since $G-M$ is not a complete graph, it follows from Lemma 7 that $M$ does not have three vertices that have the same neighbourhood in $G$. Hence, by Lemmas 5 and 6, the minimum degree of the graph $G-M-S$ is at least 3 .

Now, for $n \geq 8$

$$
\begin{aligned}
e(G) & =e(G-M-S)+2 m+2 s-2 \\
& \geq \frac{1}{2}(3(n-m-s))+2 m+2 s-2 \\
& =\frac{1}{2}(3 n+m+s-4) \\
& \geq \frac{3 n-2}{2}, \text { since } s \geq 2 .
\end{aligned}
$$

Subcase 2.2.2. $G-S$ is nonhamiltonian:

Then $G-S$ is MNH (as shown above); hence it follows from Theorem 1 , that $e(G-S) \geq \frac{3}{2}(n-s)$ for $n-s \geq 6$.

Thus, for $n-s \geq 6$ and $n \geq 8$

$$
\begin{aligned}
e(G) & =e(G-S)+2 s-2 \\
& \geq \frac{1}{2}(3(n-s))+2 s-2 \\
& =\frac{1}{2}(3 n+s-4) \\
& \geq \frac{3 n-2}{2}, \text { since } s \geq 2 .
\end{aligned}
$$

From [7] we have

$$
e(G-S) \geq \begin{cases}6 & \text { for } n-s=5 \\ 4 & \text { for } n-s=4\end{cases}
$$

Thus

$$
e(G) \geq \begin{cases}12 & \text { for } n=9 \text { and } n-s=5 \\ 10 & \text { for } n=8 \text { and } n-s=5 \text { or } n-s=4 .\end{cases}
$$


The smallest MNH graphs $F_{4}$ and $F_{5}$ of order 4 and 5 respectively, are depicted in Fig. 2; cf. [7]. The graphs $G_{8}$ and $G_{9}$ (see Fig. 3) are obtained, respectively, by using $F_{4}$ with $s=4$ or $F_{5}$ with $s=3$, and $F_{5}$ with $s=4$.

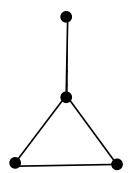

$\mathrm{F}_{4}$

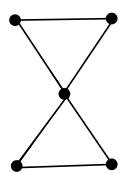

$\mathrm{F}_{5}$

Fig. 2

CASE 3. $\langle S\rangle$ has exactly one component, say $H$ :

Since

$$
\sum_{v \in S} d_{G}(v)=3 s-2, \text { for } s=1,2
$$

it follows that

$$
\begin{aligned}
e(G) & =e(G-M)+2 m \\
& =\frac{1}{2}\left(\sum_{v \in V(G-M)-S} d_{G-M}(v)+\sum_{v \in S} d_{G-M}(v)\right)+2 m \\
& \geq \frac{1}{2}(3(n-m-s)+3 s-2)+2 m \\
& =\frac{1}{2}(3 n+m-2) \\
& \geq \frac{3 n-2}{2} .
\end{aligned}
$$

From the previous theorem we have $g(8)=10, g(9)=12$ and $g(n) \geq\left\lceil\frac{3 n-2}{2}\right\rceil$ for $n \geq 10$. The MNT graphs $G_{n}$ of order $n$ with $g(n)$ edges, for $n \leq 9$ are given in Fig. 3 .
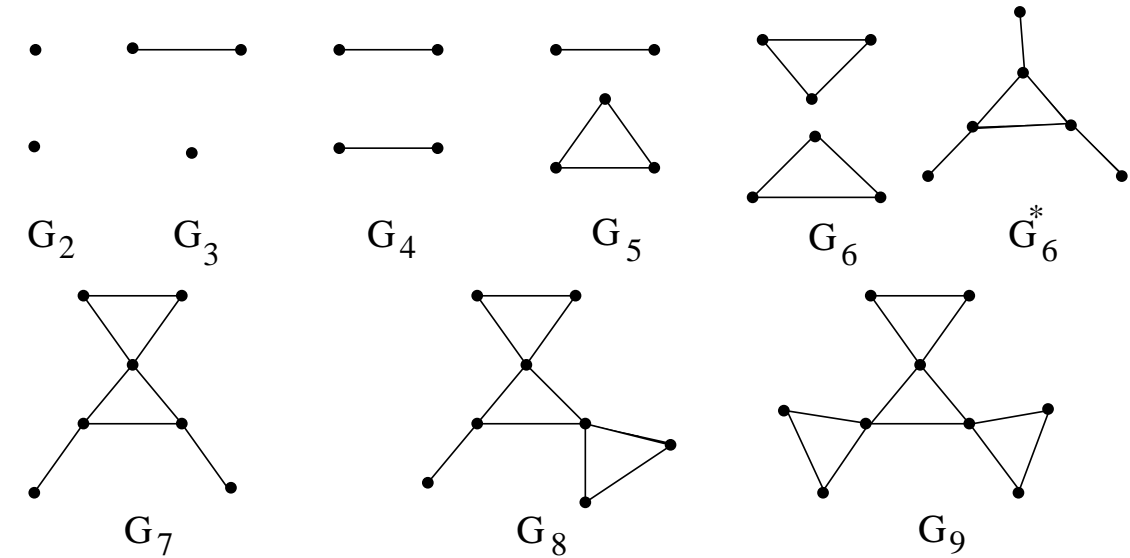

Fig. 3 
In [5] Dudek, Katona and Wojda constructed, for every $n \geq 54$ as well as for every $n \in I=\{22,23,30,31,38,39,40,41,42,43,46,47,48,49,50,51\}$, a MNT graph of size $\left\lceil\frac{3 n-2}{2}\right\rceil$ in the following way: Consider a cubic MNH graph $G$ with the property that

(1) there is an edge $y_{1} y_{2}$ of $G$, such that $N\left(y_{1}\right) \cap N\left(y_{2}\right)=\emptyset$, and

(2) $G+e$ has a hamiltonian cycle containing $y_{1} y_{2}$ for every $e \in E(\bar{G})$.

Now take two graphs $H_{1}$ and $H_{2}$, with $H_{1} \cong K_{1}$ and $H_{2} \cong K_{1}$ or $H_{2} \cong K_{2}$ and join each vertex of $H_{i}$ to $y_{i} ; i=1,2$. The new graph is a MNT graph of order $v(G)+2$ and size $e(G)+2$ or of order $v(G)+3$ and size $e(G)+4$.

It follows from results in [3] and [4] that for every even $n \geq 52$ as well as for $n \in$ $\{20,28,36,38,40,44,46,48\}$ there exists a cubic MNH graph of order $n$ that satisfies (1) and (2). Thus this construction provides MNT graphs of order $n$ and size $\left\lceil\frac{3 n-2}{2}\right\rceil$ for every $n \geq 54$ as well as for every $n \in I$.

We determined, by using the Graph Manipulation Package developed by Siqinfu and Sheng Bau*, that the Petersen graph also satisfies the above property. Hence, according to the above construction, there are also MNT graphs of order $n$ and size $\left\lceil\frac{3 n-2}{2}\right\rceil$ for $n=12,13$.

Thus $g(n)=\left\lceil\frac{3 n-2}{2}\right\rceil$ for $n \geq 54$ as well as for every $n \in I \cup\{12,13\}$.

It remains an open problem to find $g(n)$ for $n=10,11$ and those values of $n$ between 13 and 54 which are not in $I$.

*Acknowledgement We wish to thank Sheng Bau for allowing us the use of the programme, Graph Manipulation Package Version 1.0 (1996), Siqinfu and Sheng Bau, Inner Mongolia Institute of Finance and Economics, Huhhot, CN-010051, People's Republic of China.

\section{References}

[1] B. Bollobás, Extremal graph theory, London: Academic Press (1978).

[2] J.A. Bondy, Variations on the hamiltonian theme, Canad. Math. Bull. 15 (1972), 57-62.

[3] L. Clark and R. Entringer, Smallest maximally nonhamiltonian graphs, Period. Math. Hung. 14 (1983), 57-68.

[4] L.H. Clark, R.C. Entringer and H.D. Shapiro, Smallest maximally nonhamiltonian graphs II, Graphs and Combin. 8 (1992), 225-231.

[5] A. Dudek, G.Y. Katona and A.P. Wojda, Hamiltonian Path Saturated Graphs with Small Size. Submitted

[6] M. Frick and J. Singleton, Cubic maximal nontraceable graphs. Submitted.

[7] X. Lin, W. Jiang, C. Zhang and Y. Yang, On smallest maximally nonhamiltonian graphs, Ars Combin. 45 (1997), 263-270.

[8] R.C. Read and R.J. Wilson, An Atlas of Graphs, Oxford Science Publications, Oxford University Press (1998).

[9] B. Zelinka, Graphs maximal with respect to absence of hamiltonian paths, Discussiones Mathematicae. Graph Theory 18 (1998), 205-208. 